\title{
EFICIENCIA DE LOS MÉTODOS DIAGNÓSTICOS EN EL ESTUDIO DEL SANGRADO UTERINO ANORMAL EN LA PERI Y POSTMENOPAUSIA
}

\author{
Pablo Sanhueza R., Luis Oliva P. \\ Departamento de Obstetricia y Ginecología, Escuela de Medicina, Facultad de Ciencias de la Salud, Universidad \\ Andrés Bello.
}

\section{RESUMEN}

El sangrado uterino anormal es un importante síntoma que puede indicar la presencia de cáncer o hiperplasia endometrial. El objetivo de este estudio es evaluar la eficiencia de los diversos métodos diagnósticos en pacientes peri y postmenopáusicas con sangrado uterino anormal y proponer una vía de manejo en estas pacientes. Diferentes métodos diagnósticos serán evaluados, incluyendo ecografía transvaginal, sonohisterografía, Doppler, resonancia magnética nuclear, biopsia por Pipelle, dilatación y curetage e histeroscopia. Está demostrado que la ecografía transvaginal tiene una alta eficiencia para el reconocimiento de lesiones difusas, mientras que sonohisterografía tiene una alta eficiencia para identificar lesiones focales. Por otro lado, la biopsia por Pipelle ha demostrado descartar patología difusa con una alta eficiencia. Se concluye que la mejor vía para el estudio de pacientes con sangrado uterino anormal es realizar una ecografía transvaginal y una histerosonografía en el mismo momento, seguido por una biopsia mediante Pipelle. Finalmente, puede ser sugerido que por esta vía es altamente probable que todos los casos de cáncer e hiperplasia endometrial puedan ser identificados.

\section{PALABRAS CLAVES: Sangrado uterino anormal, metrorragia, cáncer endometrial}

\section{SUMMARY}

Abnormal uterine bleeding is an important symptom that can mean presence of endometrial cancer or endometrial hyperplasia. The aim of this study is to assess the effectiveness of selected diagnostic methods in peri and potmenopausal women with abnormal uterine bleeding, and to propose a way to manage in these patients. Different diagnostic methods are assessed, which include transvaginal ultrasonography, sonohysterography, Doppler technique, magnetic resonance images, biopsy by Pipelle, dilatation and curettage, and hysteroscopy. It is founded that ultrasonography has a high efficiency when it comes for identifying diffuse lesions, while sonohysterography has a high efficiency for identify focal lesions. On the other hand biopsy by Pipelle has demonstrated to rule out diffuse pathology with a high efficiency. It is concluded that the best way to manage patients with abnormal uterine bleeding by performing ultrasonography and sonohysterography at the same time followed by a Pipelle biopsy. Finally, it can be suggested that by this way it is highly probable that all cases with endometrial carcinoma and endometrial hyperplasia can be identified.

KEY WORDS: Abnormal uterine bleeding, metrorrhagia, endometrial cancer 


\section{INTRODUCCIÓN}

El sangrado uterino anormal puede manifestarse en diferentes formas, involucrando menorragia, metrorragia o goteo (spotting). Este síntoma es considerado una de las causas de consulta más frecuente en ginecología.

En mujeres perimenopáusicas la mayoría de las causas de sangrado uterino anormal están relacionadas con el cese de la ovulación y en menor grado con cáncer e hiperplasia endometrial. En pacientes postmenopáusicas, es particularmente importante debido a que puede significar la presencia de cáncer endometrial, siendo el principal síntoma en el 75 a $90 \%$ de las pacientes que sufren esa enfermedad. Más importante aún, 5 a $10 \%$ de todas las pacientes postmenopáusicas con sangrado uterino anormal tienen cáncer endometrial, y esta cifra podría alcanzar el $60 \%$ en las pacientes de 80 años (1).

En mujeres peri y postmenopáusicas el sangrado uterino anormal puede significar la presencia de 2 tipos de lesiones de la cavidad uterina. El primer tipo, más frecuente en pacientes postmenopáusicas, son las lesiones difusas dentro de las cuales destacan la hiperplasia endometrial y el cáncer endometrial. La hiperplasia endometrial corresponde a una lesión pre maligna la cual puede convertirse en cáncer endometrial en el $1 \%$ a $29 \%$ de los casos dependiendo de la estructura y citología de la lesión (2). El segundo tipo, más frecuente en pacientes perimenopáusicas, son las lesiones focales dentro de las cuales destacan los pólipos y los miomas, y en la mayoría de los casos no poseen un potencial maligno.

El problema para los ginecólogos, es cómo identificar el origen del sangrado uterino anormal con una alta efectividad para descartar cáncer endometrial y por la vía menos invasiva posible. En este sentido, la mayoría de los estudios evalúan los diversos métodos diagnósticos con el análisis histológico del útero cuando este es analizado en forma posterior a una histerectomía, por lo tanto claramente este procedimiento es considerado lo mejor. Sin embargo, no es posible proponer este tipo de estudio en todas las pacientes con sangrado uterino anormal debido a que la mayoría de ellas no tienen cáncer endometrial. Más aún, la histerectomía está relacionada con complicaciones quirúrgicas e involucra un alto costo. Con el objeto de evaluar cuál es el mejor procedimiento diagnóstico muchos estudios han sido desarrollados, sin embargo, pocos de estos son concluyentes en determinar cuál es la mejor vía de manejo en estas pacientes y hasta hoy en día no existe consenso entre los ginecólogos. El objetivo de este estudio es evaluar la eficiencia de diferentes métodos diagnósticos en pacientes peri y postmeno- páusicas con sangrado uterino anormal, y proponer una vía de manejo en estas pacientes.

\section{MÉTODOS BASADOS EN IMÁGENES}

Ecografía transvaginal. Es uno de los más importantes métodos para evaluar los órganos pélvicos. A través de esta técnica se puede evaluar el endometrio y por esta vía medir su grosor. Es una técnica económica, sin complicaciones, reproducible y ampliamente conocida entre los ginecólogos.

Debido a que las lesiones difusas pueden incrementar el grosor endometrial, muchos estudios han establecido una relación entre el grosor de este tejido y el riesgo de cáncer endometrial o hiperplasia endometrial. Además, la ecografía transvaginal permite la evaluación de si el endometrio es heterogéneo o hiperecogénico, las cuales, ambas características han sido relacionadas con el riesgo de cáncer endometrial independiente de su grosor.

Esta observación a permitido a los ginecólogos determinar un grosor endometrial crítico de $5 \mathrm{~mm}$ en pacientes postmenopáusicas. Un reciente estudio demostró que si el grosor endometrial es mayor de $5 \mathrm{~mm}$, el riesgo de cáncer endometrial es de 7,3\%, y disminuye si el grosor endometrial es menor o igual a $5 \mathrm{~mm}$. Además, si el grosor endometrial es igual o menor de $5 \mathrm{~mm}$, esto tiene una sensibilidad de $92 \%$ y una especificidad de $81 \%$ para identificar cáncer endometrial; estos resultados caen a $66 \%$ de sensibilidad y $79 \%$ de especificidad si este es de $10 \mathrm{~mm}$ (3). Esto significa que mientras menor grosor endometrial sea considerado como normal más cánceres endometriales serán identificados, y que mientras mayor grosor sea considerado como normal, más cánceres endometriales pasarán desapercibidos.

Sin embargo, la ecografía transvaginal tiene sus limitaciones. Un reciente meta-análisis estableció que el grosor endometrial medido por ecografía transvaginal tenía una limitada predicción diagnóstica para cáncer e hiperplasia endometrial después de evaluar 51 estudios que fueron llevados a cabo sobre 9031 pacientes, y que el cáncer endometrial puede ocurrir en pacientes con grosor endometrial menor de $5 \mathrm{~mm}$ y considerado como normal (4). Además, un estudio demostró que la correlación entre el grosor endometrial cuando este es medido por ecografía transvaginal y cuando este es medido in vivo posterior a una histerectomía, es sólo correcta en el $65 \%$ de los casos (5)

En resumen, a pesar de sus limitaciones la ecografía transvaginal ha demostrado ser uno de los métodos más valiosos para evaluar el sangrado uterino anormal en pacientes peri y postmenopáusicas, teniendo especial valor debido a que es rápido, fácil, 
económico y reproducible, permitiendo en la mayoría de los casos descartar cáncer e hiperplasia endometrial con una alta sensibilidad y especificidad.

Sonohisterografía. Es una técnica derivada de la ecografía transvaginal y consiste en introducir una solución fisiológica mediante una sonda a través del cuello uterino dilatando la cavidad endometrial. Por esta vía, y a través de la visualización transvaginal, es posible ver directamente la cavidad identificando las lesiones existentes en ella.

Muchos estudios han demostrado que la sonohisterografía tiene una alta correlación para identificar patología endometrial, con especial énfasis en diagnosticar lesiones focales. En un reciente estudio llevado a cabo en pacientes peri y postmenopáusicas, fue demostrado que la sonohisterografía tiene una sensibilidad de $94,1 \%$ y una especificidad de $88,5 \%$ para identificar todo tipo de patología endometrial, y que es practicable en el $97 \%$ de las pacientes con sangrado uterino anormal (6).

Sin embargo, este procedimiento diagnóstico tiene sus limitaciones. En un reciente estudio que fue llevado a cabo en pacientes peri y postmenopáusicas fue demostrado que la histerosonografía tiene una alta sensibilidad y especificidad identificando lesiones focales, tales como pólipos y miomas, con valores de $94,1 \%$ y $84,5 \%$ respectivamente, y que tiene una baja correlación para identificar lesiones difusas, tales como cáncer e hiperplasia endometrial, con una sensibilidad de 33,3\% (7). Este aspecto es crucial, debido a que lo más importante para un ginecólogo es descartar hiperplasia y cáncer endometrial en pacientes con sangrado uterino anormal. Además, hay pocos estudios demostrando los beneficiosos aspectos de la histerosonografía, cuando esta es aplicada sólo en pacientes postmenopáusicas. Más aún, la técnica de introducir un catéter dentro de la cavidad uterina podría ser riesgosa y difícil de hacer en pacientes postmenopáusicas, existiendo el riesgo teórico de diseminar células endometriales cancerosas de estar presente la lesión.

En conclusión, las mayores virtudes y contribuciones de la histerosonografía en pacientes con sangrado uterino anormal es identificar lesiones focales, y que es una económica, fácil y reproducible técnica diagnóstica.

Doppler. Teóricamente en un proceso tumoral, tal como en hiperplasia o cáncer endometrial, la resistencia al flujo sanguíneo de los vasos disminuye. Por lo tanto existe una relación entre la resistencia de los vasos endometriales y la posibilidad de que este tejido tenga un componente maligno. A pesar de los aspectos teóricos y considerando de que existe un estudio demostrando una reducción de la resistencia de los vasos endometriales en pacientes con lesio- nes difusas, la contribución de la evaluación Doppler no mejora significativamente ni la sensibilidad ni la especificidad en la detección de hiperplasia o cáncer endometrial, cuando éste es comparado con la medición endometrial realizada por ecografía transvaginal (8). Por lo tanto, la evaluación Doppler de la cavidad endometrial no tiene mayor contribución en el estudio de las pacientes peri y postmenopáusicas con sangrado uterino anormal.

Resonancia magnética nuclear. La resonancia magnética nuclear es una técnica basada en la propiedad que tienen los núcleos de los átomos contenidos en los tejidos lo cual permite estudiar la estructura de los órganos, tales como el endometrio. Debido a que la resonancia magnética involucra una alta tecnología es que existen altos costos asociados. Actualmente se considera que la resonancia magnética nuclear juega un pequeño rol detectando lesiones difusas, y hoy en día es usada solamente para establecer un plan de tratamiento en pacientes que han sido diagnosticadas con cáncer endometrial (9). Por lo tanto, a pesar de que en sus inicios se atribuyó un rol en el estudio de pacientes con sangrado uterino anormal, actualmente la resonancia magnética nuclear no tiene ninguna contribución en el proceso diagnóstico en estas pacientes.

\section{MÉTODOS BASADOS EN BIOPSIAS}

Biopsia por Pipelle. Es un valioso procedimiento y permite identificar cáncer o hiperplasia endometrial en pacientes con sangrado uterino anormal. Corresponde a un económico, fácil y reproducible procedimiento, el cual es llevado a cabo en la consulta, no necesitando mayor infraestructura.

En un reciente meta-análisis fue demostrado que para identificar cáncer endometrial, la biopsia tomada por Pipelle tiene una sensibilidad y una especificidad de $95,3 \%$ y $81 \%$ respectivamente (10). Aunque sólo un $4 \%$ de la cavidad endometrial es legrada por el pequeño dispositivo, la biopsia por Pipelle ha demostrado tener una alta correlación entre el diagnóstico histológico realizado a través de éste y el análisis realizado posterior a una histerectomía (11). Sin embargo, otro estudio no demostró estos buenos resultados (12). Además, un estudio demostró que la muestra obtenida por Pipelle podría ser considerada inadecuada en el $20 \%$ de los casos (13). Más aún, la biopsia por Pipelle es considerada un inadecuado método en pacientes con sangrado uterino anormal que tienen lesiones focales, debido a que pólipos y miomas no podrían ser tomadas por el pequeño dispositivo y podrían pasar desapercibidas.

En resumen, a pesar de sus limitaciones, la biopsia obtenida por Pipelle ha demostrado ser un 
útil procedimiento permitiendo descartar patología difusa con una alta eficiencia en la mayoría de los casos.

Dilatación y curetaje. Por muchos años dilatación y curetaje (D\&C) había sido considerado el estándar dorado para estudiar las pacientes con sangrado uterino anormal, debido a que era el único procedimiento que podía hacer el correcto diagnóstico en pacientes peri y postmenopáusicas con una alta sensibilidad y efectividad. De hecho, alcanzando el sitio donde el sangrado se está produciendo y tomando una muestra o la mayor parte del endometrio, es considerado hasta hoy, por algunos, la mejor técnica para estudiar la cavidad endometrial. Más aún, como estándar dorado, la mayoría de los estudios desarrollados durante las últimas décadas tenían que compararse con D\&C para demostrar su efectividad en pacientes con sangrado uterino anormal.

Aunque hoy en día algunos ginecólogos consideran que $\mathrm{D} \& \mathrm{C}$ continúa siendo el mejor procedimiento diagnóstico, hay un estudio demostrando que la histeroscopía tiene mejor sensibilidad y especificidad que D\&C para identificar lesiones focales y difusas en pacientes con sangrado uterino anormal (14). Después de esto, es difícil encontrar estudios actualizados que demuestren los beneficiosos aspectos de la $D \& C$.

Adicionalmente, algunos estudios han demostrado que la $D \& C$ no es un valioso método para estudiar la cavidad en pacientes con sangrado uterino anormal debido a que sólo un $60 \%$ de la cavidad uterina es legrada (15). Más aún, se demostró que en el $16 \%$ de las pacientes sólo fue legrado el $25 \%$ de la cavidad y la mayoría de las lesiones focales pasan desapercibidas en un procedimiento normal (11). Finalmente y relacionado con lo anterior, se ha determinado que la tasa de falsos negativos es entre 10 y $60 \%$ para identificar cáncer endometrial $(1,16)$.

Debido a que D\&C es un procedimiento quirúrgico, costoso y no sin complicaciones, es difícil de proponer en todas las pacientes con sangrado uterino anormal. Además, hoy en día D\&C no es considerado el estándar dorado, por lo que no hay suficientes argumentos para indicar este procedimiento invasivo en todas las pacientes con sangrado uterino anormal.

\section{MÉTODO BASADO EN LA VISUALIZACIÓN DE LA CAVIDAD UTERINA}

Histeroscopia. Desde que hubo un estudio demostrando que la histeroscopia es mejor que la D\&C en el diagnóstico de pacientes con sangrado uterino anormal (16), los ginecólogos han considerado que este es el mejor método para la evaluación de pacientes con este síntoma. Estos buenos resultados han sido confirmados por posteriores estudios los cuales han demostrado sensibilidades de $96 \%$ para el diagnóstico de hiperplasia endometrial y de $92 \%$ para el diagnóstico de pólipos $(17,18)$. Sin embargo, algunos investigadores consideran que la histeroscopía tendría poco valor en la detección de lesiones difusas, tales como cáncer o hiperplasia endometrial $(11,15)$. A esto se suma que otros estudios han mostrado que la histeroscopia tiene limitaciones en el estudio de las lesiones difusas, con sensibilidad de 22,2\% para el diagnóstico de hiperplasia endometrial (7). A pesar del hecho de que hoy en día la histeroscopía es considerada el estándar dorado en el estudio de pacientes con sangrado uterino anormal, podrían existir casos de cáncer e hiperplasia endometrial que pasarían desapercibidos cuando esta técnica es usada. Por lo tanto, este método tiene limitada capacidad en el diagnóstico de lesiones difusas y su uso es inadecuado en estos casos. Más aún, así como D\&C, es un procedimiento quirúrgico costoso, y no exento de complicaciones.

\section{DISCUSIÓN}

El sangrado uterino anormal es uno de los síntomas más frecuentes en pacientes peri y postmenopáusicas. Este síntoma puede significar la presencia de hiperplasia y cáncer endometrial en hasta el $60 \%$ de las pacientes con edades superiores a los 80 años. Sin embargo, la mayoría de las pacientes que presentan este síntoma no tienen estas patologías, por lo tanto es altamente necesario establecer cuál es la mejor vía de manejo en ellas. Por otro lado, el desafío para los ginecólogos es descartar estas condiciones premalignas y malignas con una alta eficacia y por la vía menos invasiva posible. Esta revisión ha evaluado la eficiencia de los métodos diagnósticos utilizados en pacientes peri y postmenopáusicas con sangrado uterino anormal para descartar lesiones difusas, tales como hiperplasia y cáncer endometrial.

Un gran número de estudios han sido desarrollados. La mayoría de ellos comparan dos métodos separadamente y pocos de ellos corresponden a un meta-análisis. Con respecto a la ecografía transvaginal, éste es un procedimiento ampliamente disponible y sin complicaciones. Además, tiene una alta sensibilidad y especificidad para la detección de lesiones difusas. Por otro lado, histerosonografía es altamente efectiva en la detección de lesiones focales y comparte las características beneficiosas de la ecografía transvaginal. 
Con respecto a los métodos basados en biopsias, la biopsia por Pipelle ha demostrado ser un útil procedimiento, permitiendo descartar lesiones difusas con alta eficacia. D\&C en el pasado y actualmente la histeroscopía han sido considerados ser los mejores métodos para evaluar la cavidad endometrial. Sin embargo, la histeroscopía está relacionada con complicaciones quirúrgicas, y es necesaria experiencia y un intenso entrenamiento. Además, ésta tiene un alto valor para identificar lesiones focales, pero tiene un valor limitado en la detección de lesiones difusas. Finalmente, debido a que la mayoría de las pacientes con sangrado uterino anormal no tienen patología endometrial, es que es imposible proponer hacer este procedimiento costoso e invasivo en todas estas pacientes.

A pesar de el hecho de que esta revisión no realizó ni una comparación ni un meta-análisis entre los diferentes procedimientos, ha sido demostrado que es desaconsejable proponer una vía de manejo en estas pacientes sólo con un método diagnóstico. Esto, debido a que ninguno de los procedimientos tiene una sensibilidad del $100 \%$, por lo tanto muchos casos de cáncer endometrial podrían pasar desapercibidos.

Por lo tanto, puede ser inferido que la mejor vía de manejo en estas pacientes es hacer una combinación de métodos diagnósticos con el fin de lograr altas sensibilidades y especificidades en el proceso diagnóstico. En este sentido, es propuesto hacer la medición del grosor endometrial mediante una ecografía transvaginal más la realización de una histerosonografía en el mismo momento. Estos procedimientos deben ser seguidos por la toma de una biopsia por Pipelle. Con esta vía de manejo, los ginecólogos podrían ser capaces de descartar patologías difusas y focales con una alta eficacia. Consecuentemente, es altamente probable que todos los casos de cáncer e hiperplasia endometrial puedan ser identificados.

Debido a que no existen estudios comparativos, prospectivos y randomizados, que evalúen las diferentes vías de manejo en estas pacientes, es que son necesarios estudios con estas características. Estos deben ser realizados incluyendo algunos procedimientos diagnósticos simultáneos con el fin de establecer cuál es la mejor vía de manejo para el diagnóstico del sangrado uterino anormal en la peri y postmenopausia.

\section{BIBLIOGRAFÍA}

1. Symonds I. Ultrasound, hysteroscopy and endometrial biopsy in the investigation of endometrial cancer. Best Pract Res Clin Obstet Gynaecol 2001;15(3):381-91.

2. Garg R, del Carmen M. Endometrial hyperplasia: diagnosis and management. Postgraduate Obstet Gynecol 2005;25(1):1-5.

3. Smith-Bindman R, Weiss E, Feldstein V. How thick is too thick? When endometrial thickness should prompt biopsy in postmenopausal women without vaginal bleeding. Ultrasound Obstet Gynecol 2004;5:558-65.

4. Gupta J, Chien P, Voit D, Clark J, Khan K. Ultrasonographic endometrial thickness for diagnosing endometrial pathology in women with postmenopausal bleeding: a meta-analysis. Acta Obstet Gynecol Scand 2002;81:799-816.

5. Saha T, Amer S, Biss J, Thakare H, Williams S, FarreII T, Calvert J. The validity of transvaginal ultrasound measurement of endometrial thickness: a comparison of ultrasound measurement with direct anatomical measurement. Br J Obstet Gynaecol 2004;111:141924.

6. Pasrija S, Trivedi S, Narula M. Prospective study of saline infusion sonohysterography in evaluation of perimenopausal and postmenopausal women with abnormal uterine bleeding. J Obstet Gynaecol 2004;30(1):27-33.

7. Krampl $\mathrm{E}$, Bourne $\mathrm{T}$, Hurlen-Solbakken $\mathrm{H}$, Istre $\mathrm{O}$. Transvaginal ultrasonography sonohysterography and operative hysteroscopy for the evaluation of abnormal uterine bleeding. Acta Obstet Gynecol Scand 2001;80:616-22.

8. Arslan M, Erdem A, Erdem M, Yazici G, Himmetoglu O, Gursoy R. Transvaginal color Doppler ultrasonography for prediction of pre-cancerous endometrial lesions. Int J Gynecol Obstet 2003;80:299-306.

9. Manfredi R, Gui B, Maresca G, Fanfani F, Bonomo L. Endometrial cancer: magnetic resonance imaging. Abdominal Imaging 2005;30:626-36.

10. Dijkhuizen F, Mol B, Brojmann $\mathrm{H}$. The accuracy of endometrial sampling in the diagnosis of patients with endometrial carcinoma and hyperplasia. A metaanalysis. Cancer 2000;89:1765-72.

11. Critchley $H$, Warner $P$, Lee A, Brechin S, Guise J, Graham B. Evaluation of abnormal uterine bleeding: comparison of three outpatient procedures within cohorts defined by age and menopausal status. Health Technol Assess 2004;8(34):iii-iv,1-158.

12. Ferry J, Farnsworth A, Webster M, Wren B. The efficacy of endometrial biopsy in detecting endometrial cancer. Aust N Z J Obstet Gynaecol 1993;33:76-8.

13. Farrel T, Jones N, Owen P, Baird A. The significance of an "insufficient" Pipelle sample in the investigation of post-menopausal bleeding. Acta Obstet Gynecol Scand 1991;78:810-12.

14. Gimpelson R, Rappold H. A comparative study between panoramic hysteroscopy with directed biopsies and dilatation and curettage. A review of 276 cases. Am J Obstet Gynecol 1998;158:489-92.

15. Hatasaka $\mathrm{H}$. The evaluation of abnormal uterine bleeding. Clin Obstet Gynecol 2005;48(2):258-73.

16. Al-Kamil R. Clinical effectiveness of hysteroscopy in abnormal uterine bleeding. J Obstet Gynaecol 2001;21(6):614-6.

17. Van Dongen $\mathrm{H}$, de Kroon $\mathrm{C}$, Jacobi C, Trimbos J, Jansena F. Diagnostic hysteroscopy in abnormal uterine bleeding: a systematic review and meta-analysis. $\mathrm{Br} \mathrm{J}$ Obstet Gynaecol 2007;114:664-75.

18. Lalchandani S, Phillips K. Evaluation of endometrial cavity-investigation options. Rev Gynaecol Pract 2003;3:165-70. 